\title{
Continuous Tensor Product States which are Translation Invariant but not Quasi-Free
}

\author{
A. Guichardet \\ Faculté des Sciences, Poitiers, France \\ A. WULFSOHN \\ University of California, Berkeley, Calif.
}

Received August 23, 1969; in revised form January 19, 1970

\begin{abstract}
We show how the theory of continuous tensor products can be used to construct, for commutation relations, translation invariant but not quasi-free states as continuous tensor products of states for systems with one degree of freedom.
\end{abstract}

\section{Introduction}

As was shown by R. T. Powers in [6] $\S 5.3$ for the case of anticommutation relations, all translation invariant states which can be constructed as infinite tensor products of states for systems with a finite number of degrees of freedom are quasi-free and consequently not very interesting for physical applications; in this paper we show how the theory of continuous tensor products allows us to construct, in the case of commutation relations, translation invariant but not quasi-free states as continuous tensor products of states for systems with one degree of freedom; we consider only the nonrelativistic case since, unfortunately, we are not able to carry out the same construction in the relativistic case.

\section{§1. The Algebras Associated with a Real Symplectic Space}

We consider a real symplectic space $(E, \sigma)$, i.e. a real vector space $E$ with a non-degenerate symplectic form $\sigma$; we call representation of $(E, \sigma)$ every mapping $U$ of $E$ into the unitary operators of a complex Hilbert space such that

(i) for each $x$ in $E$ the mapping $\mathbb{R} \ni h \leftrightarrow U(h x)$ is strongly continuous

(ii) $U(x+y)=e^{i \sigma(x, y)} U(x) U(y)$.

With a real symplectic space one can associate several algebras:

1) The von Neumann algebra $\mathscr{A}_{E, \sigma}$ defined in [2], $\S 1.3$; when $E$ is finite dimensional $\mathscr{A}_{E, \sigma}$ is nothing but $\mathscr{L}(H)$ where $H$ is the space 
of the Schrödinger representation of $(E, \sigma)$; in the general case $\mathscr{A}_{E, \sigma}$ is the von Neumann inductive limit of the algebras $\mathscr{A}_{F, \sigma}$ with $F$ a finite dimensional subspace of $E$. There is a representation $W$ of $(E, \sigma)$ into $\mathscr{A}_{E, \sigma}$ which has the following universal property: given a Hilbert space $H$, the mapping $\pi \mapsto \pi \circ W$ is a bijection between the normal representations of $\mathscr{A}_{E, \sigma}$ in $H$ and the representations of $(E, \sigma)$ in $H$.

2) The Banach *-algebra $A_{E, \sigma}$ (which is similar to the algebra considered in [5]; $A_{E, \sigma}$ is the Banach space $l^{1}(E)$ whose elements are complex functions on $E$ satisfying $\sum_{x \in E}|f(x)|<\infty$, equipped with the norm

the multiplication

$$
\|f\|=\sum|f(x)|,
$$

$$
(f g)(z)=\sum_{x+y=z} e^{-i \sigma(x, y)} f(x) g(y)
$$

and the involution

$$
f^{*}(x)=\overline{f(-x)}
$$

we denote by $\delta_{x}$ the unitary element of $A_{E, \sigma}$ defined by

then

$$
\delta_{x}(y)=\left\{\begin{array}{l}
1 \text { if } y=x \\
0 \text { if } y \neq x
\end{array}\right.
$$

$$
\delta_{x+y}=e^{i \sigma(x, y)} \delta_{x} \delta_{y}
$$

given a Hilbert space $H$, the mapping $\pi \mapsto \pi \circ \delta$ is a bijection between the representations of $A_{E, \sigma}$ in $H$ such that $h \mapsto \pi\left(\delta_{h x}\right)$ is strongly continuous for each $x \in E$ and the representations of $(E, \sigma)$ in $H$. In particular there exists a unique morphism $T: A_{E, \sigma} \mapsto \mathscr{A}_{E, \sigma}$ such that the diagramm

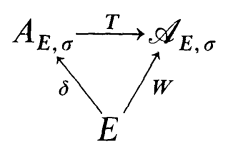

is commutative; $\operatorname{Im} T$ is strongly dense in $\mathscr{A}_{E, \sigma}$.

Concerning the states of $\mathscr{A}_{E, \sigma}$ and $A_{E, \sigma}$ there are bijective correspondences between

a) the complex functions $\psi$ on $E$ satisfying the following conditions

$-\psi(O)=1$

$-\sum_{n, p} c_{n} \bar{c}_{p} e^{i \sigma\left(x_{n}, x_{p}\right)} \psi\left(x_{n}-x_{p}\right) \geqq 0 \quad \forall c_{1}, \ldots c_{m} \in \mathbb{C}, \quad x_{1}, \ldots x_{m} \in E$

- for each $x \in E$ the mapping $\mathbb{R} \quad h \rightarrow \psi(h x)$ is continuous; such a function $\psi$ will be called a generating functional;

b) the normal states $\varphi$ of $\mathscr{A}_{E, \sigma}$;

c) the states $\chi$ of $A_{E, \sigma}$ satisfying: for each $x \in E$ the mapping $h \mapsto \chi\left(\delta_{h x}\right)$ is continuous.

These correspondences are given by $\psi=\varphi \circ W=\chi \circ \delta, \chi=\varphi \circ T$. 


\section{§ 2. A Particular Case of Real Symplectic Space}

From now on we suppose $E$ is a complex vector space of complex functions on $T=\mathbb{R}^{n}$ which are continuous and with compact support; and we set

$$
\sigma(x, y)=\operatorname{Im}(x \mid y)=\operatorname{Im} \int x(t) \overline{y(t)} d t \quad \forall x, y \in E ;
$$

we also suppose $E$ is invariant under all translations. For every $t$ in $T$ we set

$$
\begin{aligned}
E_{t} & =\mathbb{C}, \\
\sigma_{t}(\alpha, \beta) & =\operatorname{Im} \alpha \bar{\beta} \quad \forall \alpha, \beta \in E_{t}, \\
\mathscr{A}_{t} & =\mathscr{A}_{E_{t}, \sigma_{t}}, \\
W_{t} & =\text { the canonical mapping } E_{t} \rightarrow \mathscr{A}_{t}, \\
A_{t} & =A_{E_{t}, \sigma_{t}} .
\end{aligned}
$$

$\left(\left(E_{t}, \sigma_{t}\right)\right.$ is the symplectic space corresponding to a system with one degree of freedom.)

Proposition 1. $A_{E, \sigma}$ is isomorphic to the continuous tensor product of the algebras $A_{t}$; more precisely we have $A_{E, \sigma} \sim \widehat{\bigotimes}_{t \in T}^{\Gamma} A_{t}$ where $\Gamma$ is the set of all families $t \mapsto \lambda(t) \delta_{x(t)} \in A_{t}$ with $\lambda \in C_{0} \cap L^{1}+1$ and $x \in E$.

(We use the notations and definitions of [2], Ch. 3.)

First one must prove that $\left(\left(A_{t}\right)_{t \in T}, \Gamma\right)$ is a continuous family of Banach *-algebras in the sense of [2], $\S 3.4$; the proof of the axiom (iii) of [2], $\S 3.2$ is very similar to that of [3], prop. 12 ; the proof of the other axioms is trivial. Now the construction of the isomorphism is similar to that in [3], Prop. 12; we only emphasize the fact that this isomorphism $F$ carries each element $\delta_{x} \in A_{E, \sigma}$ with $x \in E$, into the element $\otimes \delta_{x(t)} \in \hat{\otimes}^{\Gamma} A_{t}$; we also recall that for each $\lambda$ in $\mathscr{C}_{0} \cap L^{1}+1$,

$$
\otimes \lambda(t) \cdot \delta_{x(t)}=\Pi \lambda(t) \cdot \otimes \delta_{x(t)}
$$

in particular if $x, y \in E$ :

$$
\begin{aligned}
\otimes \delta_{x(t)} \cdot \otimes \delta_{y(t)} & =\otimes \delta_{x(t)} \delta_{y(t)} \\
& =\otimes e^{-i x(t) \overline{y(t)}} \delta_{x(t)+y(t)} \\
& =\Pi e^{-i x(t) \overline{y(t)}} \otimes \delta_{x(t)+y(t)} \\
& =e^{-i \sigma(x, y)} \otimes \delta_{x(t)+y(t)} \\
F^{-1}\left(\otimes \delta_{x(t)} \cdot \otimes \delta_{y(t)}\right) & =e^{-i \sigma(x, y)} \delta_{x+y}=\delta_{x} \delta_{y} . \quad \text { QED. }
\end{aligned}
$$


Another Algebra Associated with $(E, \sigma)$

As explained in [2], $\S 3.6$ we can also construct the continuous tensor product $\underset{t \in T}{\bigotimes} \Gamma^{\Gamma^{\prime}} \mathscr{A}_{t}$ where $\Gamma^{\prime}$ is the set of all families $t \mapsto \lambda(t) \cdot W_{t}(x(t))$ with $\lambda \in \mathscr{C}_{0} \cap L^{1}+1$ and $x \in E$; we denote it by $\mathscr{A}_{E, \sigma}^{\prime}$ and set

$$
W^{\prime}(x)=\otimes W_{t}(x(t)) \quad \forall x \in E ;
$$

we have

$$
W^{\prime}(x+y)=e^{i \sigma(x, y)} W^{\prime}(x) W^{\prime}(y) ;
$$

moreover there is a morphism $S: \mathscr{A}_{E, \sigma}^{\prime} \rightarrow \mathscr{A}_{E, \sigma}$ such that the diagramm

is commutative.

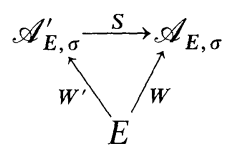

Automorphisms of the Above Algebras Induced by Translations

Every element $\tau$ of $T$ determines an automorphism of $(E, \sigma)$ :

$$
x \mapsto x_{\tau} \quad \text { with } x_{\tau}(t)=x(t-\tau) ;
$$

this automorphism determines in turn, as easily seen, automorphisms $\alpha_{\tau}, \beta_{\tau}, \gamma_{\tau}$ of $\mathscr{A}_{E, \sigma}, A_{E, \sigma}, \mathscr{A}_{E, \sigma}^{\prime}$ respectively, such that

$$
\begin{aligned}
\alpha_{\tau}(W(x)) & =W\left(x_{\tau}\right) \\
\beta_{\tau}\left(\delta_{x}\right) & =\delta_{x_{\tau}} \\
\gamma_{\tau}\left(W^{\prime}(x)\right) & =W^{\prime}\left(x_{\tau}\right) ;
\end{aligned}
$$

recalling that $A_{E, \sigma}$ and $\mathscr{A}_{E, \sigma}^{\prime}$ are continuous tensor products, $\beta_{\tau}$ and $\gamma_{\tau}$ take the simpler forms:

$$
\begin{aligned}
\beta_{\tau}\left(\otimes \delta_{x(t)}\right) & =\otimes \delta_{x(t-\tau)}, \\
\gamma_{\tau}\left(\otimes W_{t}(x(t))\right) & =\otimes W_{t-\tau}(x(t-\tau)) .
\end{aligned}
$$

These automorphisms are compatible with the canonical mappings $A_{E, \sigma} \rightarrow \mathscr{A}_{E, \sigma}$ and $\mathscr{A}_{E, \sigma}^{\prime} \rightarrow \mathscr{A}_{E, \sigma}$.

\section{§ 3. Continuous Tensor Products of States}

Consider a generating functional $\psi$ on $(E, \sigma)$ of the form

$$
\psi(x)=\exp \left[\int F_{t}(x(t)) d t\right]
$$


where each $F_{t}$ is a continuous complex function on $E_{t}=\mathbb{C}$ with the following properties:

(i) $F_{t}(O)=O$;

(ii) the function $\alpha \mapsto \psi_{t}(\alpha)=e^{F_{t}(\alpha)}$ is a generating functional on $\left(E_{t}{ }^{\prime} \sigma_{t}\right)$;

(iii) for every $x \in E$ the function $t \mapsto F_{t}(x(t))$ belongs to $\mathscr{C}_{0} \cap L^{1}$.

The state $\chi$ of $A_{E, \sigma}$ associated with $\psi$ is the continuous tensor product of the states $\chi_{t}$ (of $A_{t}$ ) associated with $\psi_{t}$; in fact for $x \in E$

$$
\begin{aligned}
\chi\left(\delta_{x}\right) & =\psi(x)=\exp \left[\int F_{t}(x(t)) d t\right] \\
& =\prod_{t \in T} \exp \left[F_{t}(x(t))\right]=\prod_{t \in T} \psi_{t}(x(t)) \\
& =\prod_{t \in T} \chi_{t}\left(\delta_{x(t)}\right) ;
\end{aligned}
$$

but as we know $\delta_{x}$ is identified with $\otimes \delta_{x(t)}$ (cf. Prop. 1). (There is a similar result for the state of $\mathscr{A}_{E, \sigma}^{\prime}$ associated with $\psi$ ). Moreover the representation associated with $\psi$ is a continuous tensor product in the sense of [4].

If moreover $F_{\mathrm{t}}$ is equal to some function $F$ independent of $t$, the state $\chi$ is obviously translation invariant, i.e. invariant under all the automorphisms $\beta_{\tau}$.

Examples. Let $F^{0}$ be a complex continuous function on $\mathbb{C}$ verifying

a) $F^{0}(O)=O$,

b) $\exp F^{0}$ is positive definite,

c) the function $\psi^{0}$ on $E$ defined by $\psi^{0}(x)=\exp \left[\int F^{0}(x(t)) d t\right]$ is positive definite;

set

$$
F(\alpha)=-\frac{1}{2}|\alpha|^{2}+F^{0}(\alpha) \quad \forall \alpha \in \mathbb{C} ;
$$

then conditions (i) and (iii) above are trivially satisfied; as for condition (ii), it is known and easily verified that $\alpha \mapsto \exp \left(-\frac{1}{2}|\alpha|^{2}\right)$ is a generating functional on $\left(E_{t}, \sigma_{t}\right)$ (the corresponding state is the Fock state; see also [2], § 1.5); then for every $\alpha_{1}, \ldots \alpha_{m}$ in $\mathbb{C}$, the matrix with coefficients

$$
\exp \left(i \alpha_{n} \bar{\alpha}_{p}\right) \cdot \psi_{t}\left(\alpha_{n}-\alpha_{p}\right)=\exp \left(i \alpha_{n} \bar{\alpha}_{p}-\frac{1}{2}\left|\alpha_{n}-\alpha_{p}\right|^{2}\right) \cdot \exp \left(F^{0}\left(\alpha_{n}-\alpha_{p}\right)\right)
$$

is positive since the coefficientwise product of two positive matrices is positive. Finally the same arguments prove that the function

$$
\begin{aligned}
x \rightarrow \psi(x) & =\exp \left[\int F(x(t)) d t\right] \\
& =\exp \left(-\frac{1}{2}\|x\|^{2}+\int F^{0}(x(t)) d t\right)
\end{aligned}
$$

is a generating functional on $(E, \sigma)$; we can thus construct many continuous tensor product states which are translation invariant.

In particular we can take $F^{0}$ of the following form:

$$
F^{0}(\alpha)=-u^{2}|\alpha|^{2}+i v \cdot \alpha+\int_{\mathbb{C}}\left(e^{i w \cdot \alpha}-1-\frac{i w \cdot \alpha}{1+|w|^{2}}\right) \frac{1+|w|^{2}}{|w|^{2}} d \mu(w)
$$


here $u$ is real, $v$ is complex, $\mu$ is a finite positive measure on $\mathbb{C}-O$, and

$$
v \cdot \alpha=\operatorname{Re} v \operatorname{Re} \alpha+\operatorname{Im} v \operatorname{Im} \alpha
$$

and similarly for $w \cdot \alpha$; conversely if $E$ is sufficiently large, for instance if it contains all infinitely differentiable functions with compact support, every $F^{0}$ satisfying a), b), c) is of the form (1) (see for instance [1], Ch. III).

\section{§4. Quasi-Free States}

Definitions. Given two real vector spaces $V$ and $W$ denote by $\mathscr{L}(V, W)$ the vector space of all linear mappings $V \rightarrow W$; if $W$ is a topological vector space we endow $\mathscr{L}(V, W)$ with the topology of the simple convergence; we say that a mapping $f: V \rightarrow W$ is differentiable if for each $x$ in $V$ there exists a linear mapping $f^{\prime}(x ;):. V \rightarrow W$ such that for every $y$ in $V$ :

$$
h^{-1}(f(x+h y)-f(x)) \rightarrow f^{\prime}(x ; y) \quad \text { when } h \text {, real, tends to } O \text {. }
$$

By the above procedure we can define inductively topologies on $\mathscr{L}(V, \mathbb{C})$, $\mathscr{L}(V, \mathscr{L}(V, \mathbb{C}))$, etc.; as usual $\mathscr{L}(V, \mathscr{L}(V, \mathbb{C}))$ shall be identified with the set of all bilinear mappings $V \times V \rightarrow \mathbb{C}$ and so on; we thus can speak of a mapping $f: V \rightarrow \mathbb{C}$ which is infinitely differentiable, and we have

$$
\begin{aligned}
& f^{(n)}\left(x ; y_{1}, \ldots y_{n}\right) \\
= & \lim _{h=0} h^{-1}\left[f^{(n-1)}\left(x+h y_{n} ; y_{1}, \ldots y_{n-1}\right)-f^{(n-1)}\left(x ; y_{1}, \ldots y_{n-1}\right)\right] ;
\end{aligned}
$$

moreover for every $x, y_{1}, \ldots y_{n}$ the function

$$
\left(h_{1}, \ldots h_{n}\right) \leftrightarrow f\left(x+h_{1} y_{1}+\cdots+h_{n} y_{n}\right)
$$

is infinitely differentiable and we have

$$
\begin{aligned}
\frac{\partial^{p_{1}+\cdots p_{n}}}{\partial h_{1}^{p_{1}} \cdots \partial h_{n}^{p_{n}}} & \left.f\left(x+h_{1} y_{1}+\cdots+h_{n} y_{n}\right)\right|_{h_{1}=\cdots=h_{n}=0} \\
= & f^{\left(p_{1}+\ldots+p_{n}\right)}(x ; \underbrace{y_{1}, \ldots y_{1}}_{p_{1} \text {-times }}, \ldots \underbrace{y_{n}, \ldots y_{n}}_{p_{n} \text {-times }}) .
\end{aligned}
$$

Returning to our $(E, \sigma)$ we denote by $E^{0}$ the set of all real functions in $E$; let $\psi$ be a generating functional such that $\psi \mid E^{0}$ is infinitely differentiable; denote by $U$ and $\xi$ the representation of $(E, \sigma)$ and cyclic vector determined by $\psi$ such that $\psi(x)=(U(x) \xi \mid \xi) \forall x \in E$; let $A(x)$ be the self-adjoint generator of the one-parameter group $h \mapsto U(h x)$.

Lemma 1. $A\left(x_{1}\right) \ldots A\left(x_{n}\right) \xi$ exists for every $x_{1}, \ldots x_{n}$ in $E^{0}$.

Proof. a) The domain $D$ of $A(x)$ is the set of all $\eta$ in $H$ such that the expression $h^{-1}(U(h x)-I) \eta$ has a strong limit when $h \rightarrow O$; but one can 
replace strong by weak; in fact let $D^{\prime}$ be the set of all $\eta$ such that $h^{-1}(U(h x)-I) \eta$ has a weak limit; $D^{\prime}$ is a linear subspace containing $D$; set

$$
A^{\prime} \eta=w-\lim (i h)^{-1}(U(h x)-I) \eta \text { for each } \eta \in D^{\prime} ;
$$

$A^{\prime}$ is easily seen to be a symmetric operator which extends $A(x)$; then $A^{\prime}=A(x)$ and $D^{\prime}=D$.

b) We now prove that the expression

$$
B=\left(h_{1} \ldots h_{n}\right)^{-1}\left(U\left(h_{1} x_{1}\right)-I\right) \ldots\left(U\left(h_{n} x_{n}\right)-I\right) \xi
$$

has a weak limit when $h_{1}, \ldots h_{n}$ tend to $O$. Denoting by $T$ the canonical mapping $A_{E, \sigma} \rightarrow H$ we have $\xi=T\left(\delta_{0}\right)$ and

$$
\begin{aligned}
B & =\left(h_{1} \ldots h_{n}\right)^{-1} \sum_{\substack{i_{1}<\cdots<i_{p} \\
p=0 \cdots n}}(-1)^{n-p} U\left(h_{i_{1}} x_{i_{1}}\right) \ldots U\left(h_{i_{p}} x_{i_{p}}\right) \cdot T\left(\delta_{0}\right) \\
& =\left(h_{1} \ldots h_{n}\right)^{-1} \sum(-1)^{n-p} U\left(h_{i_{1}} x_{i_{1}}+\cdots+h_{i_{p}} x_{i_{p}}\right) \cdot T\left(\delta_{0}\right) \\
& =\left(h_{1} \ldots h_{n}\right)^{-1} \sum(-1)^{n-p} T\left(\delta_{h_{i_{1}} x_{i_{1}}}+\cdots+h_{i_{p}} x_{i_{p}}\right) .
\end{aligned}
$$

Let us prove first that $\left(B \mid T\left(\delta_{y}\right)\right)$ has a limit for every $y$ in $E$; we have

$$
\begin{aligned}
\left(B \mid T\left(\delta_{y}\right)\right)= & \left(h_{1} \ldots h_{n}\right)^{-1} \sum(-1)^{n-p} \chi\left(\delta_{-y} \delta_{h_{i_{1}} x_{1_{1}}}+\cdots+h_{i_{p}} x_{t_{p}}\right) \\
= & \left(h_{1} \ldots h_{n}\right)^{-1} \sum(-1)^{n-p} \exp \left[i \sigma\left(y, h_{i_{1}} x_{i_{1}}+\cdots+h_{i_{p}} x_{i_{p}}\right)\right. \\
& \cdot \psi\left(-y+h_{i_{1}} x_{i_{1}}+\cdots\right) \\
= & \left(h_{1} \ldots h_{n}\right)^{-1} \sum(-1)^{n-p} \varphi\left(O, \ldots h_{i_{1}}, O, \ldots h_{i_{p}}, \ldots O\right)
\end{aligned}
$$

where we have set

$$
\varphi\left(h_{1}, \ldots h_{n}\right)=\exp \left[i \sigma\left(y, h_{1} x_{1}+\cdots+h_{n} x_{n}\right)\right] \cdot \psi\left(-y+h_{1} x_{1}+\cdots+h_{n} x_{n}\right) ;
$$

it is known (and easily verified) that (3) converges to

$$
\left.\frac{\partial^{n} \varphi}{\partial h_{1} \ldots \partial h_{n}}\right|_{h_{1}=\cdots=h_{n}=0} .
$$

Now to prove b) it is sufficient, since the $T\left(\delta_{y}\right)$ 's are total in $H$, to prove that $B$ is bounded; we have

$$
\begin{aligned}
\|B\|^{2} & =\left(h_{1} \ldots h_{n}\right)^{-2} \sum_{\substack{i_{1}<\cdots<i_{p} \\
j_{1}<\cdots<j_{q} \\
p, q=0, \ldots n}}(-1)^{p+q}\left(T\left(\delta_{h_{i_{1}} x_{i_{1}}+\cdots+h_{t_{p}} x_{t_{p}}}\right) \mid T\left(\delta_{h_{j_{1}} x_{J_{1}}+\cdots+h_{j_{q}} x_{J_{q}}}\right)\right) \\
& =\left(h_{1} \ldots h_{n}\right)^{-2} \sum(-1)^{p+q} \psi\left(h_{i_{1}} x_{i_{1}}+\cdots+h_{i_{p}} x_{i_{p}}-h_{j_{1}} x_{j_{1}}-\cdots-h_{j_{q}} x_{j_{q}}\right) ;
\end{aligned}
$$

writing out an expansion of the $\sum$ and using (2) one can see that the only terms which really occur contain $h_{1}^{a_{1}} \ldots h_{n}^{a_{n}}$ where $a_{1}, \ldots a_{n}$ are non zero even integers; this establishes our assertion.

10 Commun math Phys., Vol. 17 
c) By the part b) we know that $A\left(x_{n}\right) \xi$ exists; then

$$
\begin{aligned}
\underset{h_{n-1}=h_{n}=0}{w-\lim _{n-1}} h_{-1}^{-1}\left(U \left(h_{n-1}\right.\right. & \left.\left.x_{n-1}\right)-I\right) \cdot h_{n}^{-1}\left(U\left(h_{n} x_{n}\right)-I\right) \cdot \xi \\
& =\underset{h_{n-1}=0}{w-\lim _{n}} w-\lim (\text { the same expression }) \\
& =\underset{h_{n}=0}{w-\lim _{h_{n-1}=0}} h_{n-1}^{-1}\left(U\left(h_{n-1} x_{n-1}\right)-I\right) \cdot A\left(x_{n}\right) ;
\end{aligned}
$$

this proves that $A\left(x_{n-1}\right) A\left(x_{n}\right) \xi$ exists; and so on inductively. QED.

By the above lemma we may consider the multilinear forms on $E^{0}$

$$
\left(x_{1}, \ldots x_{n}\right) \mapsto\left(A\left(x_{1}\right) \ldots A\left(x_{n}\right) \xi \mid \xi\right) ;
$$

they are called Wightman distributions and denoted by $\mathscr{W}_{n}$; we have

$$
\begin{aligned}
\psi\left(h_{1} x_{1}+\cdots+h_{n} x_{n}\right) & =\left(U\left(h_{1} x_{1}+\cdots+h_{n} x_{n}\right) \xi \mid \xi\right) \\
& =\left(U\left(h_{1} x_{1}\right) \ldots U\left(h_{n} x_{n}\right) \xi \mid \xi\right)
\end{aligned}
$$

whence, by (2)

$$
\begin{aligned}
\psi^{(n)}\left(0 ; x_{1}, \ldots x_{n}\right) & =\left.\frac{\partial^{n}}{\partial h_{1} \ldots \partial h_{n}} \psi\left(h_{1} x_{1}+\cdots+h_{n} x_{n}\right)\right|_{h_{1}=\cdots=h_{n}=0} \\
& =i^{n} \mathscr{W}_{n}\left(x_{1}, \ldots x_{n}\right) .
\end{aligned}
$$

Then one defines the truncated Wightman distributions $\mathscr{W}_{n}^{T}$ by the following recurrence formulae

$$
\begin{gathered}
\mathscr{W}_{1}^{T}=\mathscr{W}_{1}, \\
\mathscr{W}_{n}\left(x_{1}, \ldots x_{n}\right)=\sum_{\mathscr{P}} \mathscr{W}_{n_{1}}^{T}\left(x_{i_{1,1}}, \ldots x_{i_{1, n_{1}}}\right) \ldots \mathscr{W}_{n r}^{T}\left(x_{i_{r, 1}}, \ldots x_{i_{r, n_{r}}}\right)
\end{gathered}
$$

where the sum is taken for all partitions $\mathscr{P}$ of the set $\{1,2, \ldots n\}$ into subsets

$$
\begin{aligned}
& i_{1,1}<i_{1,2}<\cdots<i_{1, n_{1}} \\
& \cdots \cdots \cdots \cdots \cdots \cdots \cdots \cdots \cdots \cdots \cdots \cdots \\
& i_{r, 1}<i_{r, 2}<\cdots<i_{r, n_{r}}
\end{aligned}
$$

with $n_{1}+\cdots+n_{r}=n$.

The state associated with $\psi$ is said quasi-free if $\mathscr{W}_{n}^{T}=0 \quad \forall n \geqq 3$ (cf. [7]).

Let us now suppose that $\psi$ has the form $\psi(x)=e^{\omega(x)}$ where $\omega$ is an infinitely differentiable mapping $E \rightarrow \mathbb{C}$, with $\omega(0)=0$; we have

$$
\begin{aligned}
\psi^{\prime}(x ; y) & =e^{\omega(x)} \cdot \omega^{\prime}(x ; y), \\
\psi^{\prime \prime}\left(x ; y_{1}, y_{2}\right) & =e^{\omega(x)}\left[\omega^{\prime}\left(x ; y_{1}\right) \omega^{\prime}\left(x ; y_{2}\right)+\omega^{\prime \prime}\left(x ; y_{1}, y_{2}\right)\right]
\end{aligned}
$$

and by induction

$$
\psi^{(n)}\left(x ; y_{1}, \ldots y_{n}\right)=e^{\omega(x)} \sum_{\mathscr{P}} \omega^{\left(n_{1}\right)}\left(x ; y_{i_{1,1}}, \ldots y_{i_{1, n_{1}}}\right) \ldots \omega^{\left(n_{r}\right)}\left(x ; y_{i_{r, 1}}, \ldots\right)
$$


it follows that, by (4)

$$
\mathscr{W}_{n}^{T}\left(x_{1}, \ldots x_{n}\right)=i^{n} \omega^{(n)}\left(O ; x_{1}, \ldots x_{n}\right) .
$$

Assume now that $\omega$ has the form $\omega(x)=\int F((x(t)) d t$ where $F$ is a complex function on $\mathbb{C}$ whose restriction to $\mathbb{R}$ is infinitely differentiable; then, for $x, y_{1}, \ldots y_{n} \in E^{0}$ we have

$$
\omega\left(x+h y_{1}\right)=\int F\left(x(t)+h y_{1}(t)\right) d t
$$

and by derivation under $\int$ :

$$
\omega^{\prime}\left(x ; y_{1}\right)=\left.\frac{d}{d h} \omega\left(x+h y_{1}\right)\right|_{h=0}=\int y_{1}(t) F^{\prime}(x(t)) d t ;
$$

then by induction

$$
\omega^{(n)}\left(x ; y_{1}, \ldots y_{n}\right)=\int F^{(n)}(x(t)) \cdot y_{1}(t) \ldots y_{n}(t) \cdot d t ;
$$

by (5)

$$
\mathscr{W}_{n}^{T}\left(x_{1}, \ldots x_{n}\right)=i^{-n} \cdot F^{(n)}(O) \cdot \int x_{1}(t) \ldots x_{n}(t) \cdot d t .
$$

We have thus proved the following:

Proposition 2. The state associated with a generating functional $\psi$ of the form $\psi(x)=\exp \left[\int F(x(t)) d t\right]$ with $F \mid \mathbb{R}$ infinitely differentiable, is quasi-free if and only if $F^{(n)}(O)=O \quad \forall n \geqq 3$.

Examples. We take $F(\alpha)=-\frac{1}{2}|\alpha|^{2}+F^{0}(\alpha)$ where $F^{0}$ is given by (1), and suppose that

$$
\int|w|^{n} d \mu(w)<+\infty \quad \forall n=1,2, \ldots ;
$$

if $\alpha$ is real we have, by setting $v_{1}=\operatorname{Re} v, w_{1}=\operatorname{Re} w$ :

$$
F(\alpha)=-\frac{1}{2} \alpha^{2}-u^{2} \alpha^{2}+i v_{1} \alpha+\int\left(e^{i w_{1} \alpha}-1-\frac{i w_{1} \alpha}{1+|w|^{2}}\right) \frac{1+|w|^{2}}{|w|^{2}} d \mu(w)
$$

whence, for $n \geqq 3$ :

$$
F^{(n)}(O)=i^{n} \int w_{1}^{n} \frac{1+|w|^{2}}{|w|^{2}} d \mu(w)
$$

we see that the corresponding state is not quasi-free unless $\mu$ is null.

\section{Bibliography}

1. Gelfand, I. M., Vilenkin, N. Ia.: Generalized functions, T. IV. New York: Academic Press.

2. Guichardet, A.: Algèbres d'observables associées aux relations de commutation. (A. Colin). Paris: 
3. Guichardet, A.: Produits tensoriels continus d'espaces et d'algèbres de Banach. Commun. Math. Phys. 5, 262-287 (1967).

4. - Wulfsohn, A.: Sur les produits tensoriels continus d'espaces hilbertiens. J. Funct. Anal. 2, 371-377 (1968).

5. Manuceau, J.: $C^{*}$-algèbre de relations de commutation. Ann. Inst. Poincaré 8, 139-161 (1968).

6. Powers, R. T.: Representations of the canonical anticommutation relations. Princeton University, Princeton thesis. 1967.

7. Robinson, D. W.: The ground state of the Bose gas. Commun. Math. Phys. 1, 159-174 (1965).
A. Guichardet
A. Wulfsohn
University of California
Berkeley, Calif., USA
7 Clos de la Sorbonne 